\title{
Nomogram for Predicting the Overall Survival and Cancer-Specific Survival of Patients with Occult Breast Cancer: A Population-Based Study
}

\section{Yang-Yu Huang}

Sun Yat-sen University Cancer Center

Guowei Ma ( $\nabla$ gzlmst@yeah.net )

Sun Yat-sen University Cancer Center https://orcid.org/0000-0002-5417-4941

\section{Shen-Hua Liang}

Sun Yat-sen University Cancer Center

\section{Lei-Lei Wu}

Tongji University Affiliated Shanghai Pulmonary Hospital

\section{Xuan Liu}

Sun Yat-sen University Cancer Center

\section{Research Article}

Keywords: occult breast cancer, overall survival, cancer-specific survival, nomogram , prognosis, population-based.

Posted Date: April 30th, 2021

DOI: https://doi.org/10.21203/rs.3.rs-415600/v1

License: (c) (i) This work is licensed under a Creative Commons Attribution 4.0 International License. Read Full License 


\section{Abstract}

Background: Occult breast cancer is a rare breast tumor, whose prognostic nomogram model has not been established. Thus, we aim to develop and validate a nomogram for evaluating the overall survival (OS) and cancer-specific survival (CSS) of patients with occult breast cancer.

Methods: Between 2004 and 2015, 704 eligible occult breast cancer patients were screened from the Surveillance, Epidemiology, and End Results (SEER) database using specific inclusion and exclusion criteria and then included in the surveillance. They were randomly divided into a training cohort $(n=494)$ and a validation cohort $(\mathrm{N}=210)$. Univariate and multivariate Cox analyses were performed to explore independent prognostic factors and establish two survival-related nomograms. Area under the curve (AUC), consistency index (C index), internal and external validation calibration curve, decision curve analysis (DCA), Kaplan-Meier analysis, and subgroup analysis were used to evaluate the nomogram.

Results: A total of seven variables were considered to be independent prognostic factors for overall survival (OS) and cancer-specific survival (CSS): age, chemotherapy, radiotherapy, Progesterone receptor (PR) status, $\mathrm{N}$ stage, number of lymph node examinations, and number of positive lymph nodes. In the training cohort, the OS nomogram-predicted AUC for three, five, and ten years were $0.792,0.775$, and 0.783 , respectively, while those of the CSS nomogram were $0.807,0.817$, and 0.812 , respectively. The calibration chart showed excellent agreement between the actual and the nomogram-predicted survival rates in both the training and validation cohorts. The C-index values of the OS nomogram in the training and validation cohorts were 0.762 and 0.782 , respectively, while those of the CSS nomogram were 0.786 and 0.816 , respectively. DCA and subgroup analysis proved the usefulness of nomograms.

Conclusion: The developed nomogram provided a comprehensive visual model of the risk of each prognostic factor. It can be conveniently used as a personalized prediction tool for the prognosis of occult breast cancer patients.

\section{Full Text}

This preprint is available for download as a PDF.

\section{Tables}

Table 1. Patients' detailed general information $(n=704)$ 


\section{Characteristic}

\section{Training Cohort $\quad(n=\quad$ Validation Cohort \\ 494) \\ = 210)}

(n

$\mathbf{N}$

179

261

54

386

65

40

3

245

208

1

40

Other

Marital status

Married

266

213

15

22

119

353

Unknown

Nodal stage

N1

311

85

98
$\% \quad N$

$36.2 \quad 82$

$52.8 \quad 105$

$10.9 \quad 23$

11.0

$78.1 \quad 175$

$13.2 \quad 24$

$8.1 \quad 11$

0.60

0

$49.6 \quad 103$

49.0

$42.1 \quad 88$

41.9

$0.2 \quad 2$

1.0

$8.1 \quad 17$

8.1

11.4

5.2

\section{3}

5.2

0

61.4

$43.1 \quad 77$

36.7

$3.0 \quad 4$

1.9

$4.5 \quad 11$

5.2

$24.1 \quad 42$

20.0

$71.5 \quad 157$

74.8

60.5

14.8

$17.2 \quad 31$

24.8 


\begin{tabular}{|c|c|c|c|c|}
\hline \multicolumn{5}{|c|}{ Estrogen receptor status } \\
\hline Positive & 252 & 51.0 & 106 & 50.5 \\
\hline Negative & 193 & 39.1 & 83 & 39.5 \\
\hline Others & 49 & 9.9 & 21 & 10.0 \\
\hline \multicolumn{5}{|c|}{$\begin{array}{l}\text { Progesterone receptor } \\
\text { status }\end{array}$} \\
\hline & 168 & 34.0 & 66 & 31.4 \\
\hline Neqative & 266 & 53.8 & 120 & 57.1 \\
\hline Others & 60 & 12.1 & 24 & 11.4 \\
\hline \multicolumn{5}{|l|}{ HER-2 status } \\
\hline Positive & 73 & 14.8 & 30 & 14.3 \\
\hline Negative & 158 & 32.0 & 65 & 31.0 \\
\hline Others & 263 & 53.2 & 115 & 54.8 \\
\hline \multicolumn{5}{|l|}{ Surgery } \\
\hline Lumpectomy & 65 & 13.2 & 21 & 10.0 \\
\hline Mastectomy & 148 & 30.0 & 74 & 35.2 \\
\hline None & 274 & 55.5 & 113 & 53.8 \\
\hline Unknown & 7 & 1.4 & 2 & 1.0 \\
\hline \multicolumn{5}{|l|}{ Radiation } \\
\hline Yes & 265 & 53.6 & 102 & 48.6 \\
\hline No & 229 & 46.4 & 108 & 51.4 \\
\hline \multicolumn{5}{|l|}{ Chemotherapy } \\
\hline Yes & 397 & 80.4 & 161 & 76.7 \\
\hline No/Unknown & 97 & 19.6 & 49 & 23.3 \\
\hline \multicolumn{5}{|c|}{$\begin{array}{l}\text { No. of examined lymph } \\
\text { nodes }\end{array}$} \\
\hline 0 & & & & \\
\hline $1-3$ & 12 & 2.4 & 0 & 2.9 \\
\hline $4-9$ & 72 & 14.6 & 28 & 13.3 \\
\hline 10- & 62 & 12.6 & 26 & 12.4 \\
\hline Unknown & 271 & 54.9 & 123 & 58.6 \\
\hline
\end{tabular}


No. of positive lymph nodes

0

13

1322.63

1.4

$1-3$

240

$48.6 \quad 108$

51.4

4-9

76

$15.4 \quad 29$

13.8

$10-$

53

$10.7 \quad 32$

15.2

112

$22.7 \quad 38$

18.1

Table 2. Survival analyses of overall survival for occult breast cancer patients in training cohort 


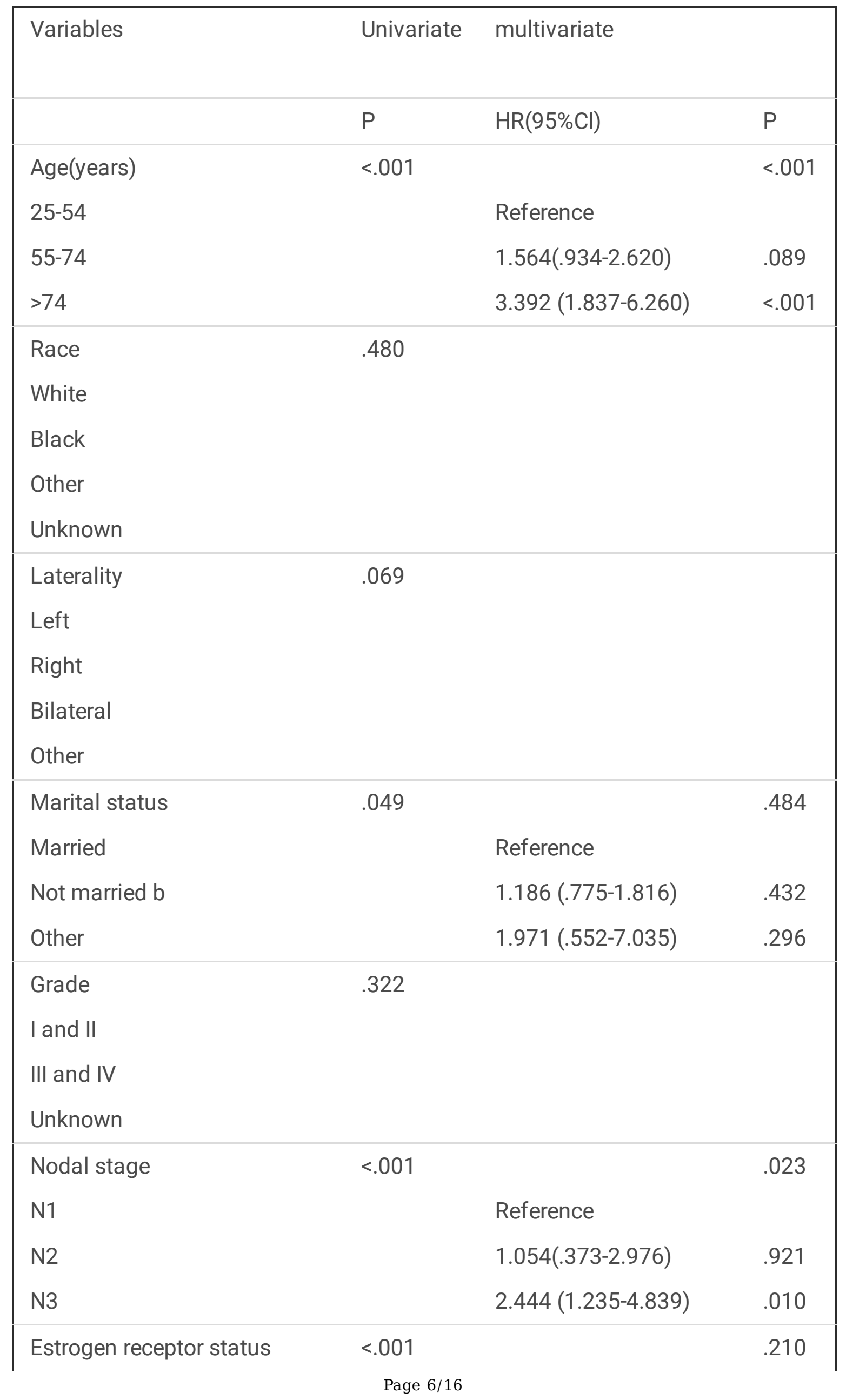




\begin{tabular}{|c|c|c|c|}
\hline Positive & & $0.660(0.381-1.143)$ & .138 \\
\hline Negative & & Reference & \\
\hline Others & & $2.434(0.300-19.772)$ & .405 \\
\hline Progesterone receptor status & .002 & & .008 \\
\hline Positive & & $0.359(0.182-0.709)$ & .003 \\
\hline Negative & & Reference & \\
\hline Others & & $0.295(.039-2.239)$ & .238 \\
\hline $\begin{array}{l}\text { HER-2 status } \\
\text { Positive } \\
\text { Negative } \\
\text { Others }\end{array}$ & .248 & & \\
\hline $\begin{array}{l}\text { Surgery } \\
\text { Lumpectomy } \\
\text { Mastectomy } \\
\text { None } \\
\text { Unknown }\end{array}$ & .234 & & \\
\hline Radiation & .003 & & .007 \\
\hline Yes & & $0.525(0.327-0.841)$ & .007 \\
\hline No & & Reference & \\
\hline Chemotherapy & $<.001$ & & .009 \\
\hline Yes & & $0.519(0.318-0.848)$ & .009 \\
\hline No/Unknown & & Reference & \\
\hline $\begin{array}{l}\text { No. of examined lymph nodes } \\
0\end{array}$ & $<.001$ & & .018 \\
\hline $1-3$ & & Reference & \\
\hline $4-9$ & & $2.709(0.649-11.307)$ & .172 \\
\hline $10-$ & & $1.276(0.260-6.269)$ & .764 \\
\hline Unknown & & $0.760(0.185-3.116)$ & .703 \\
\hline & & $0.947(0.332-2.702)$ & .918 \\
\hline No. of positive lymph nodes & $<.001$ & & .090 \\
\hline
\end{tabular}




\begin{tabular}{|lll|}
0 & & \\
$1-3$ & Reference & \\
$4-9$ & $2319.823(0-7.402 \mathrm{e}+40)$ & .860 \\
$10-$ & $8129.127(0-2.601 \mathrm{e}+41)$ & .838 \\
Unknown & $6231.362(0-1.990 \mathrm{e}+41)$ & .843 \\
& $7272.320(0-2.329 \mathrm{e}+41)$ & .840 \\
\hline
\end{tabular}

Table 3. Survival analyses of cancer-specific survival for occult breast cancer patients in training cohort 


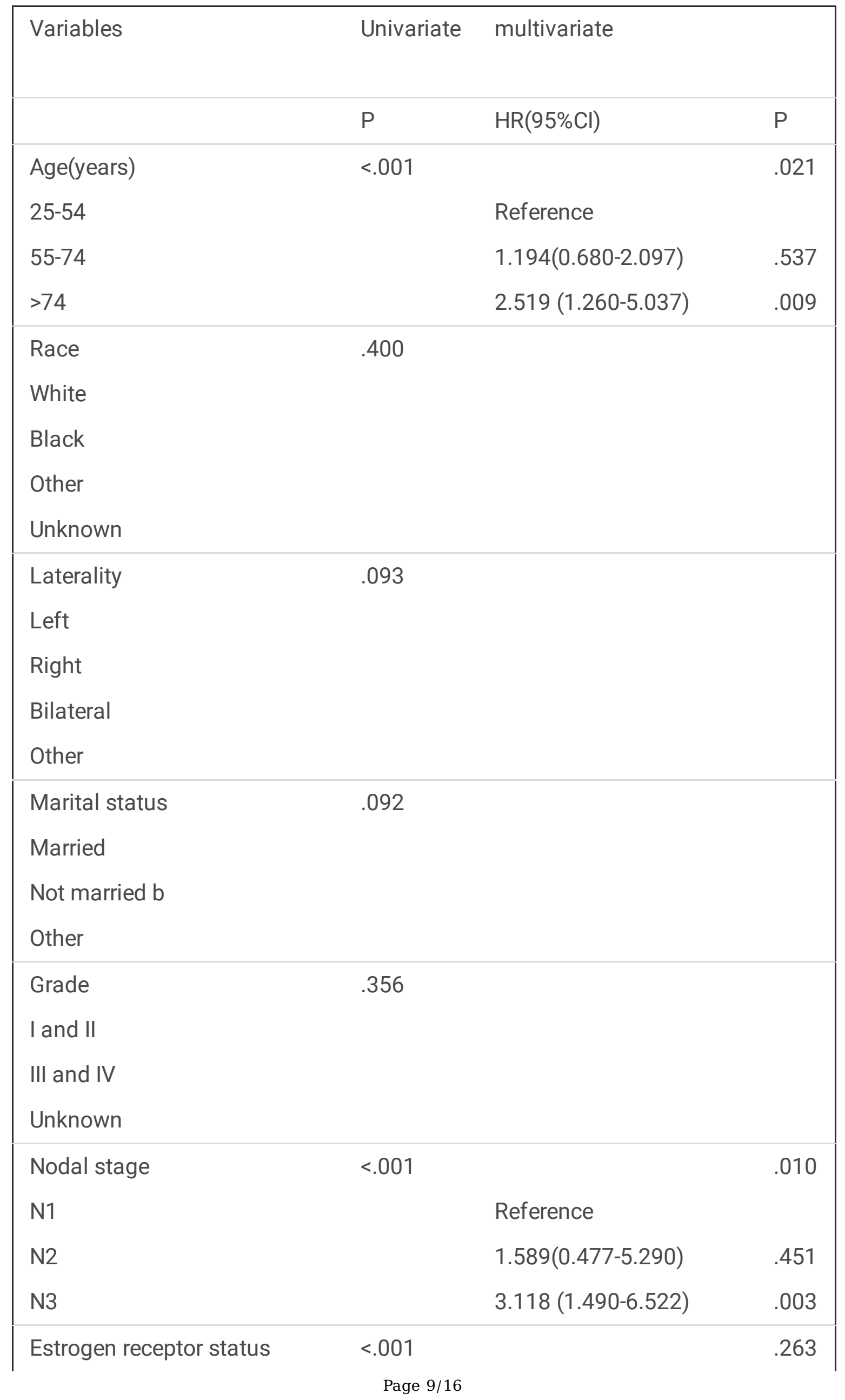




\begin{tabular}{|c|c|c|c|}
\hline \multirow{2}{*}{\multicolumn{2}{|c|}{$\begin{array}{l}\text { Positive } \\
\text { Negative }\end{array}$}} & \multirow{2}{*}{$\begin{array}{l}0.572(0.291-1.122) \\
\text { Reference }\end{array}$} & \multirow[t]{2}{*}{.104} \\
\hline & & & \\
\hline Others & & $4.206 \mathrm{e}+03(0-3.401 \mathrm{e}+44)$ & .862 \\
\hline Progesterone receptor status & .008 & & .263 \\
\hline Positive & & $0.428(0.193-0.951)$ & .104 \\
\hline Negative & & Reference & \\
\hline Others & & $0(0-1.616 \mathrm{e}+39)$ & .862 \\
\hline $\begin{array}{l}\text { HER-2 status } \\
\text { Positive } \\
\text { Negative } \\
\text { Others }\end{array}$ & .404 & & \\
\hline $\begin{array}{l}\text { Surgery } \\
\text { Lumpectomy } \\
\text { Mastectomy } \\
\text { None } \\
\text { Unknown }\end{array}$ & .267 & & \\
\hline Radiation & .013 & & .004 \\
\hline Yes & & $0.445(0.256-0.773)$ & .004 \\
\hline No & & Reference & \\
\hline Chemotherapy & .009 & & .140 \\
\hline Yes & & $0.642(0.356-1.157)$ & .140 \\
\hline No/Unknown & & Reference & \\
\hline No. of examined lymph nodes & $<.001$ & & .006 \\
\hline 0 & & Reference & \\
\hline $1-3$ & & $2.994(0.600-14.926)$ & .181 \\
\hline $4-9$ & & $2.177(0.383-12.363)$ & .380 \\
\hline $10-$ & & $0.505(0.100-2.548)$ & .408 \\
\hline Unknown & & $1.152(0.381-3.482)$ & .802 \\
\hline No. of positive lymph nodes & $<.001$ & & .026 \\
\hline 0 & & Reference & \\
\hline
\end{tabular}




\section{Figures}

Points
age
chemotherapy
radiation
PR_status
N_status
LNexamined
Total Points
3-year survival
5-year survival
10-year survival

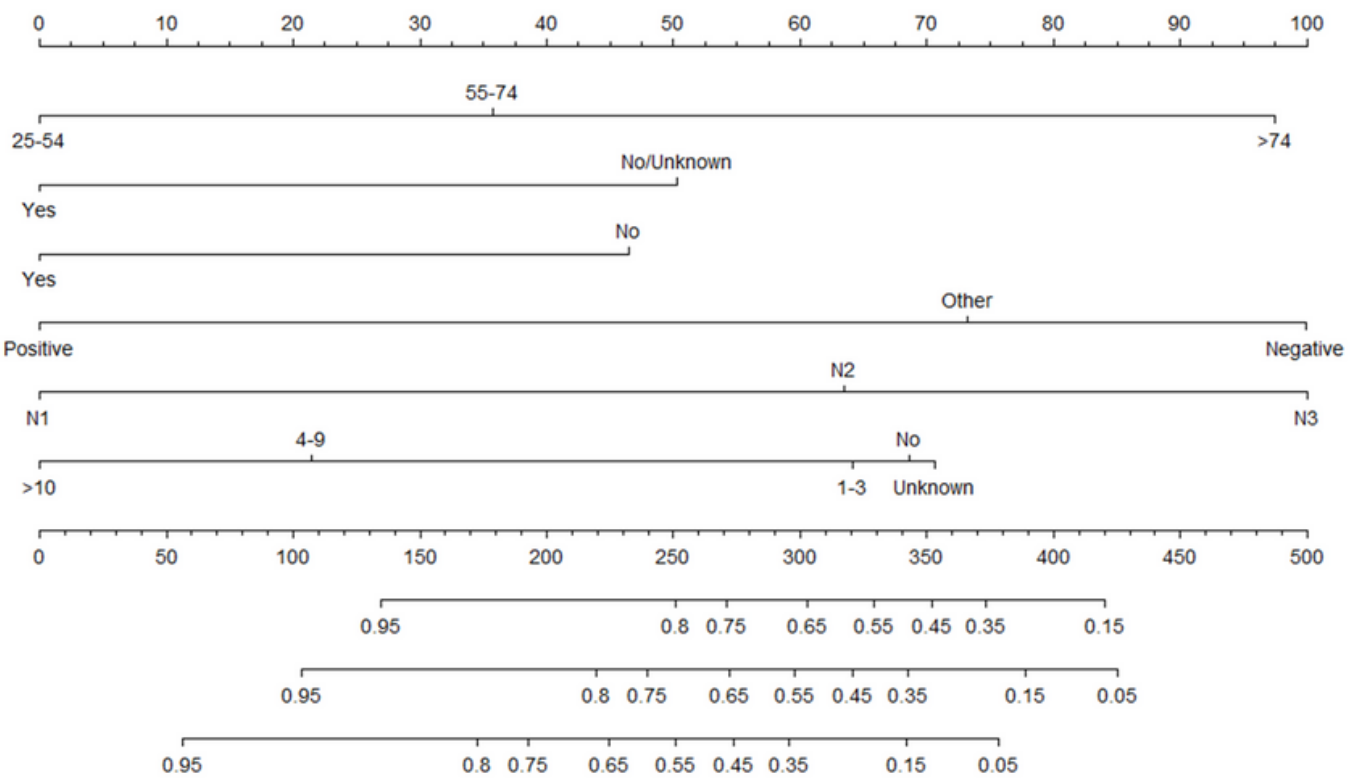

\section{Figure 1}

Nomogram predicting overall survival (OS) of occult breast cancer patients 
Points

age

radiation

LNpositive

N_status

LNexamined

Total Points

3-year survival

5-year survival

10-year survival

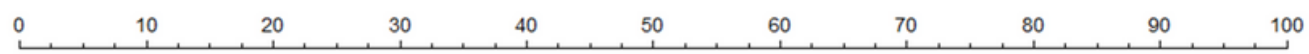

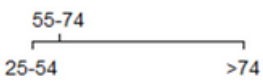

Yes

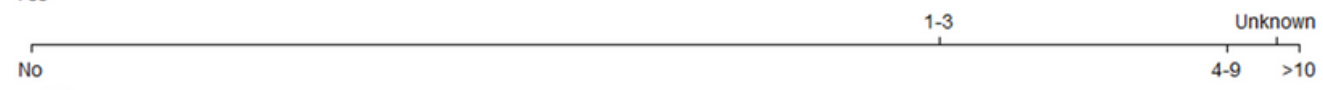

N2
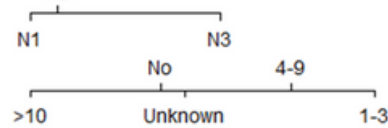

$20 \quad 40 \quad 60$

$80100 \quad 120 \quad 140 \quad 180$
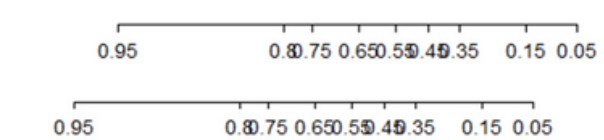

$0.95 \quad 0.80 .750 .650 .590 .49 .350 .150 .05$

\section{Figure 2}

Nomogram predicting cancer-specific survival (CSS) of occult breast cancer patients

A

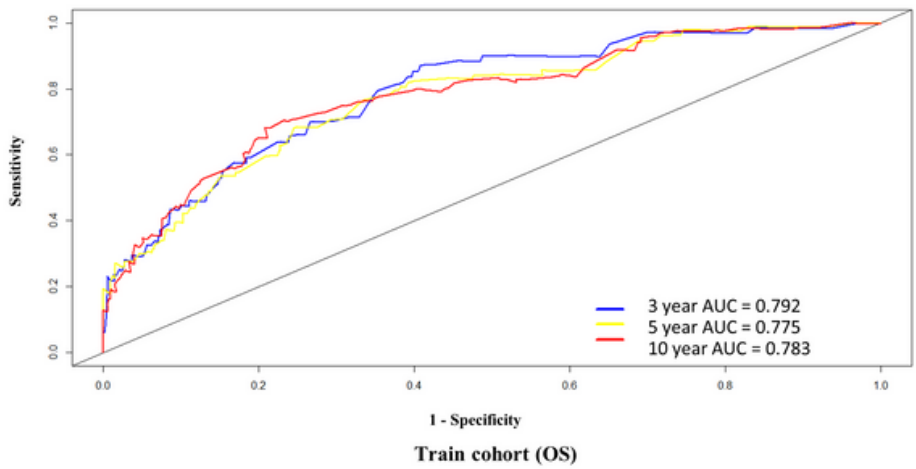

C

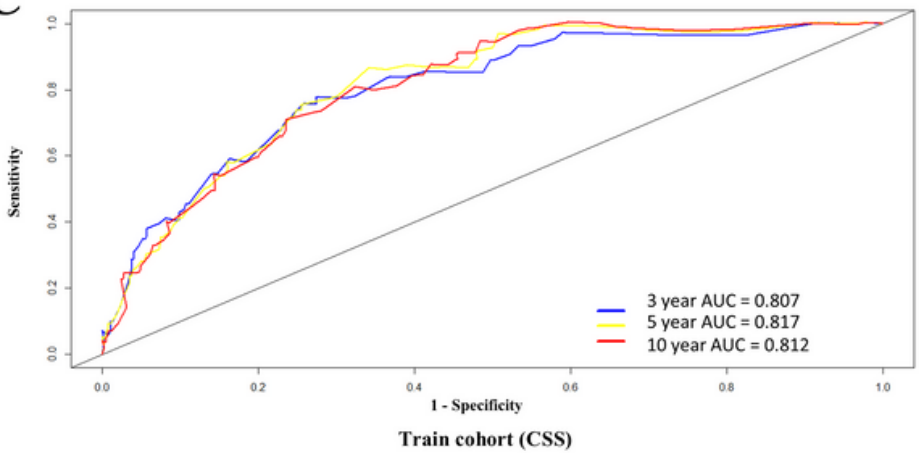

B

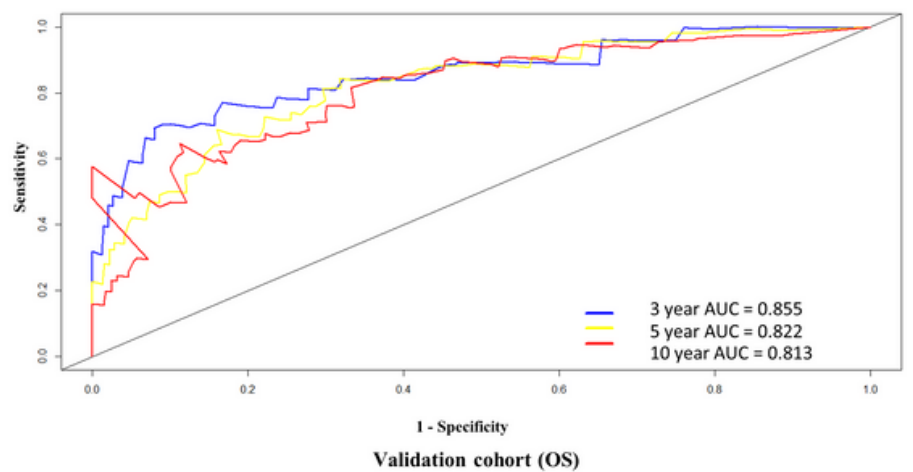

D

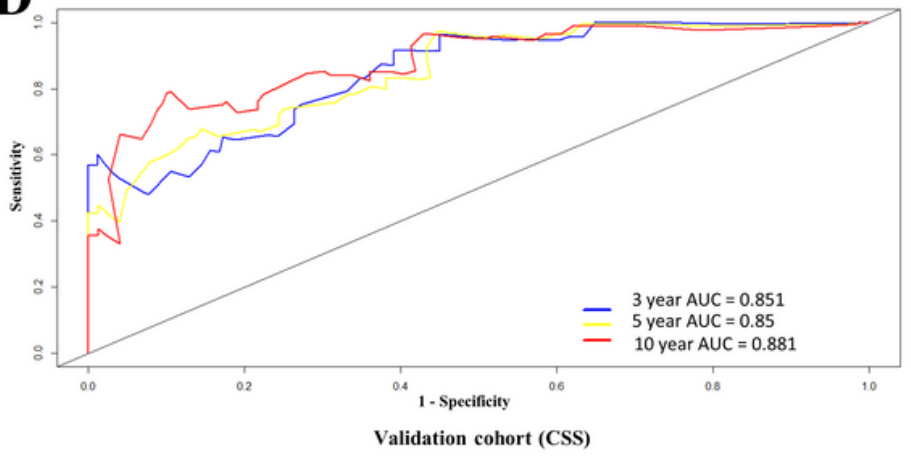


Figure 3

Performance of nomogram via receiver operating characteristic (ROC) curve

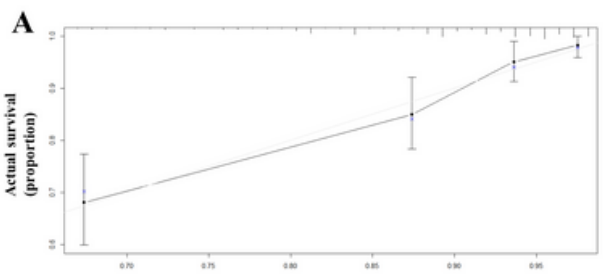

Nomogram predicted 3-Y survival(Train cohort)

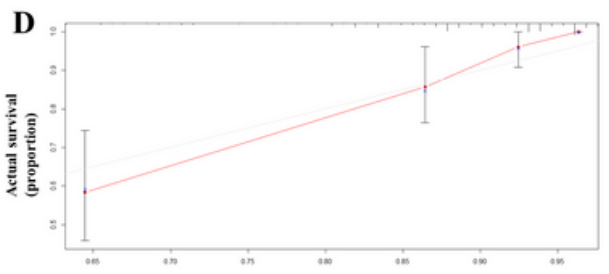

Nomogram predicted 3-Y survival(Validation cohort)

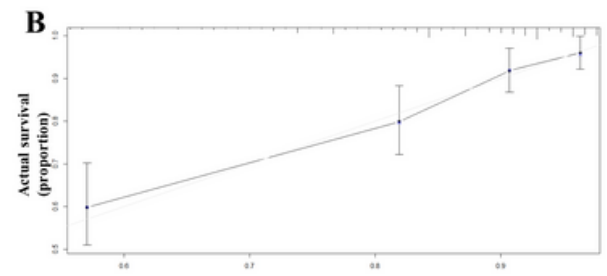

Nomogram predicted 5-Y survival(Train cohort)

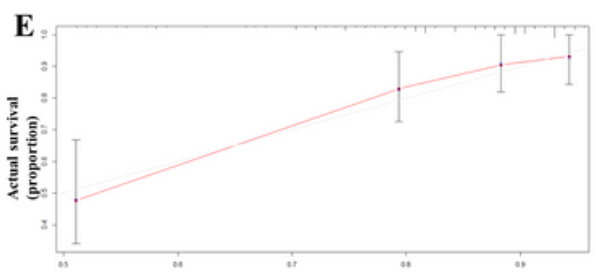

Nomogram predicted 5-Y survival(Validation cohort)

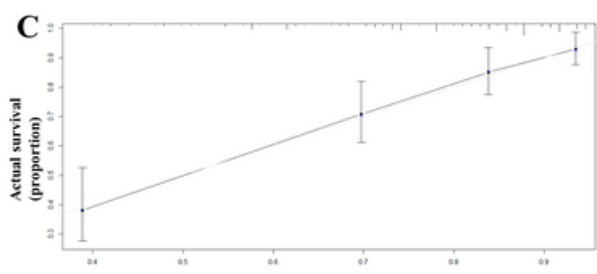

Nomogram predicted 10-Y survival(Train cohort)

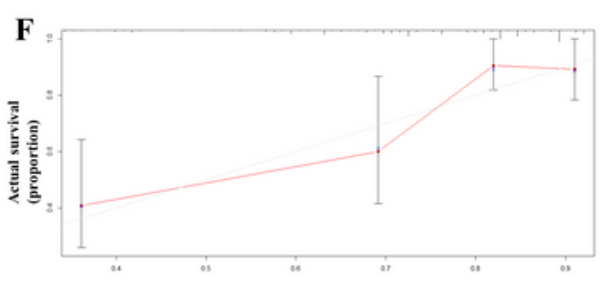

Nomogram predicted 10-Y survival(Validation cohort)

Figure 4

Internal and external calibration nomogram for overall survival (OS) 


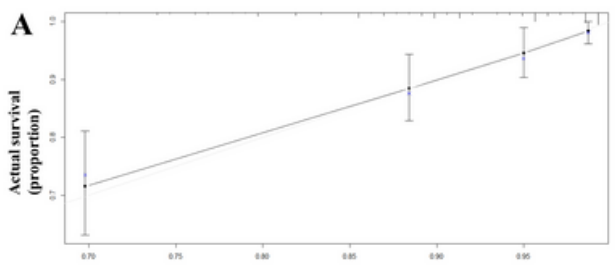

Nomogram predicted 3-Y survival(Train cohort)

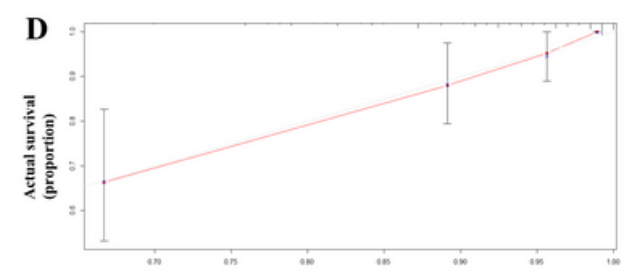

Nomogram predicted 3-Y survival(Validation cohort)

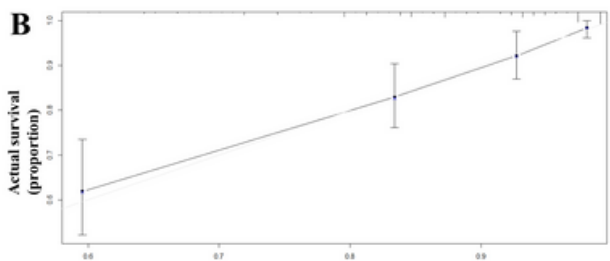

Nomogram predicted 5-Y survival(Train cohort)

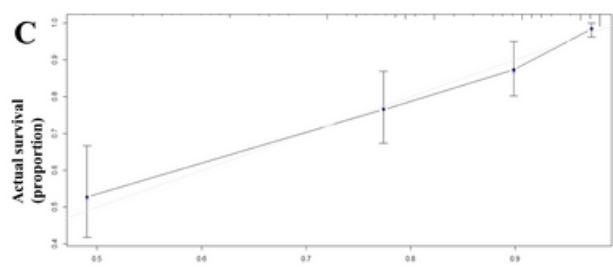

Nomogram predicted 10-Y survival(Train cohort)
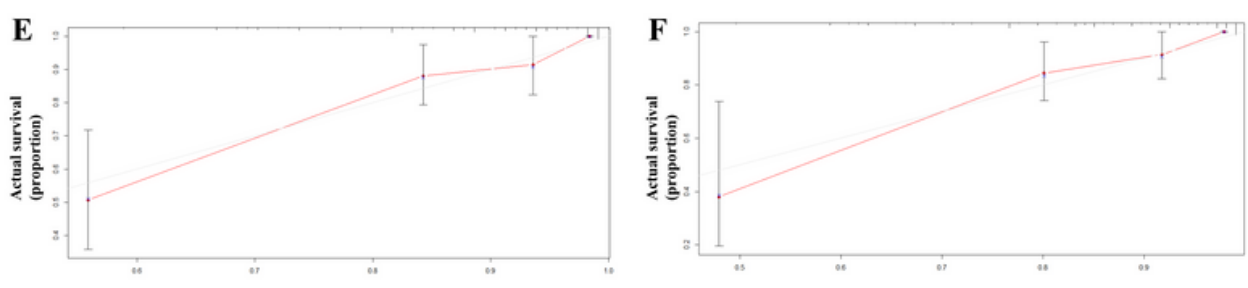

Nomogram predicted 10-Y survival(Validation cohort)

\section{Figure 5}

Internal and external calibration nomogram for cancer-specific survival (CSS)

3-Y survival(training cohort)

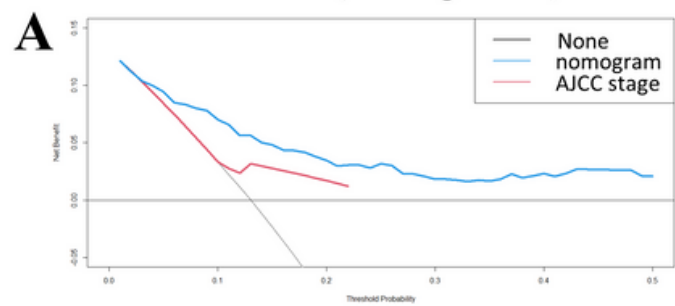

5-Y survival(training cohort)

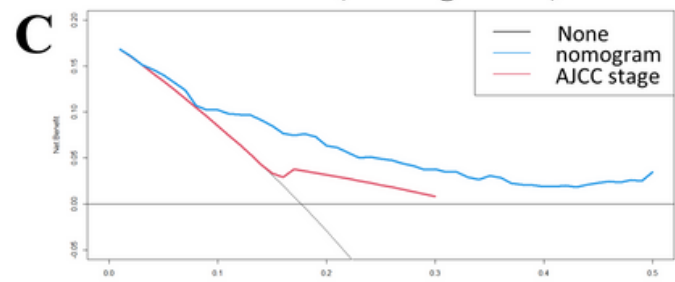

10-Y survival(training cohort)

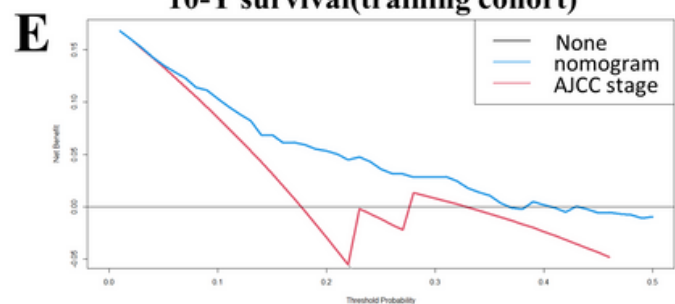

3-Y survival(validation cohort)

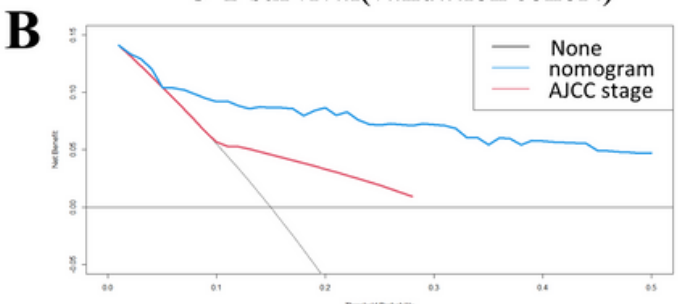

5-Y survival(validation cohort)

D

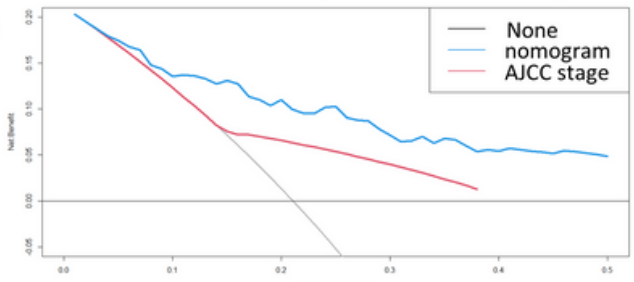

10-Y survival(validation cohort)

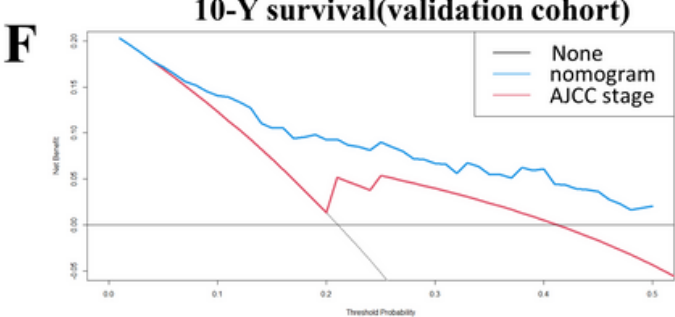


Figure 6

Decision curve analysis of the training cohort (A, C, E) and validation cohort (B, D, F) for 3403-, 5-, and 10years overall survival (OS) to compare the net benefit of the nomogram and the AJCC 341 stage.
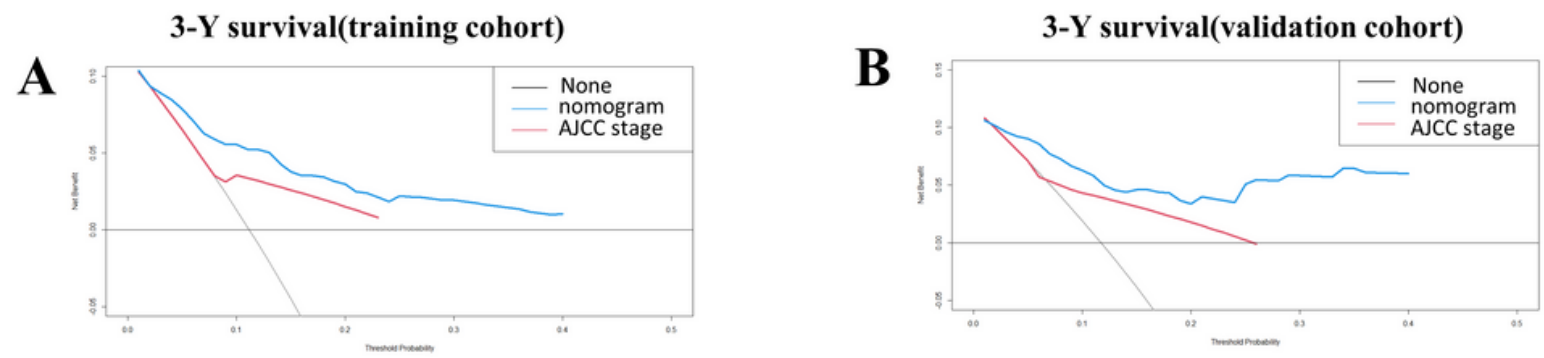

5-Y survival(training cohort)

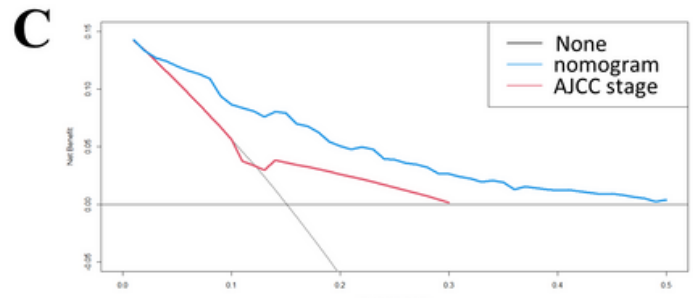

D

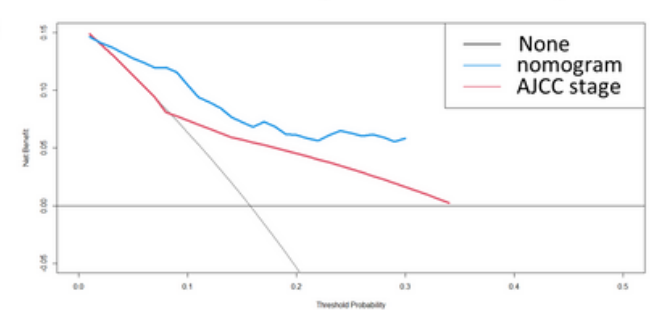

$\mathbf{E}$

10-Y survival(training cohort)

$\mathbf{F}$

10-Y survival(validation cohort)
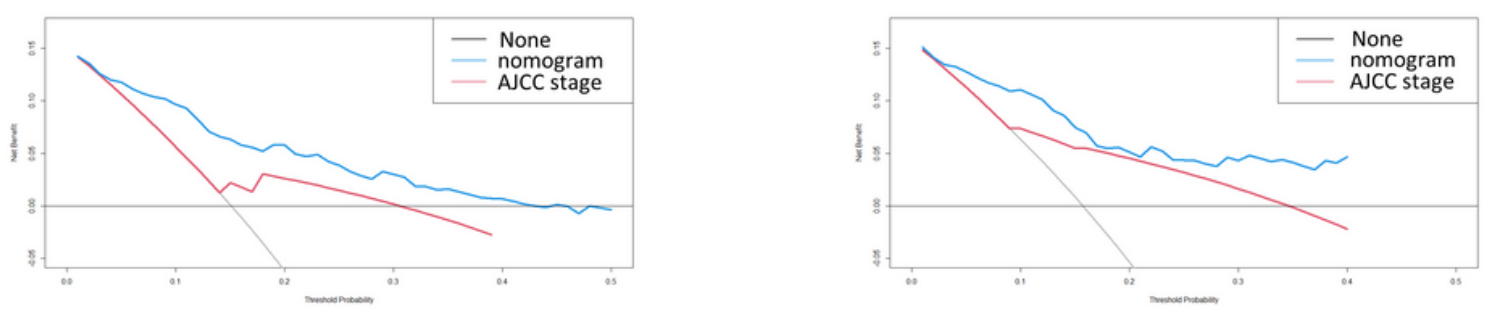

Figure 7

Decision curve analysis of the training cohort (A, C, E) and validation cohort (B, D, F) for 3443-, 5-, and 10years cancer-specific survival (CSS) to compare the net benefit of the nomogram and 345the AJCC staging. 

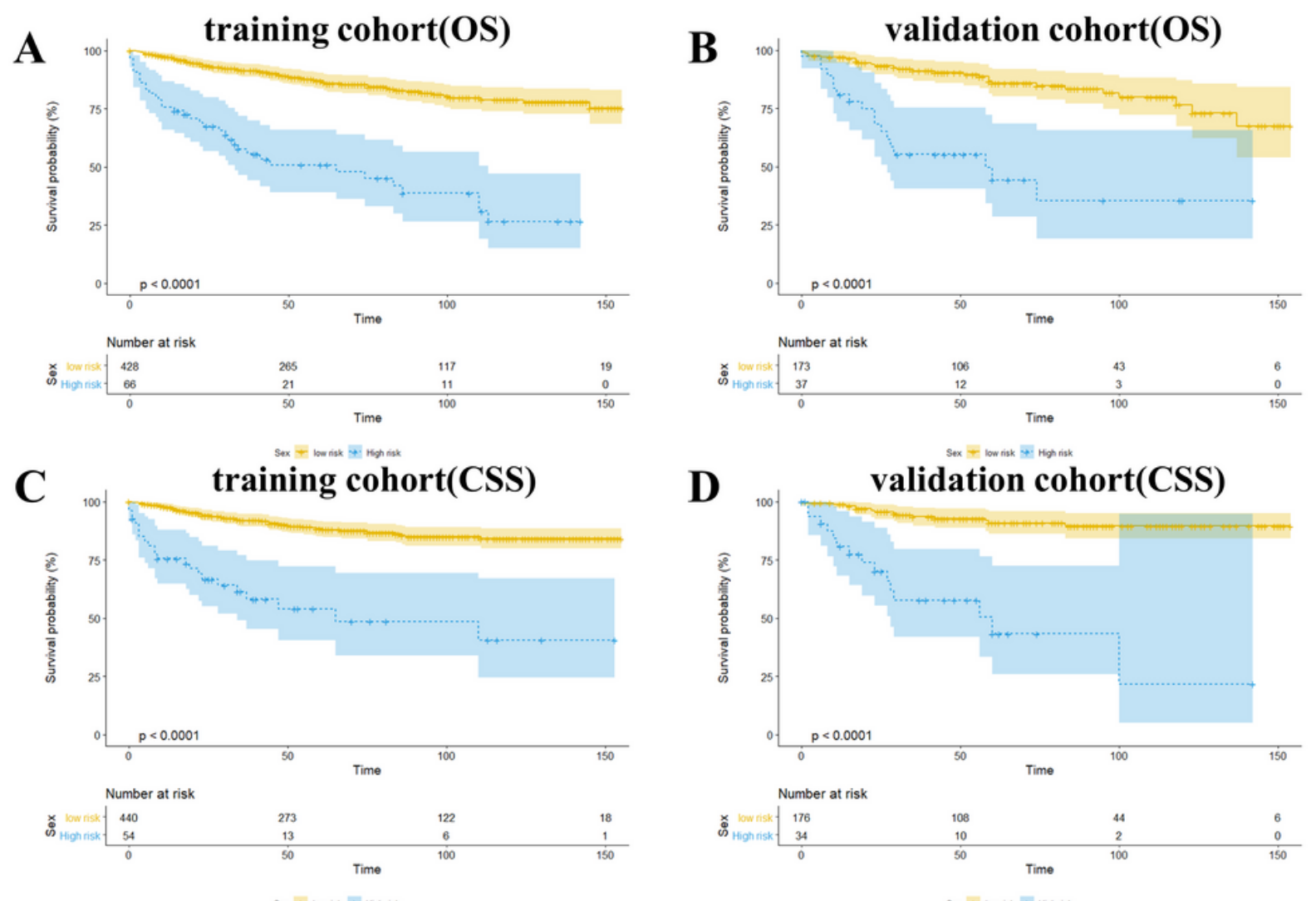

\section{Figure 8}

Survival analysis of patients after risk-stratification (A and B for overall survival (OS); C 348and D for cancer-specific survival (CSS)). 